EPJ Web of Conferences 71, 00001 (2014)

DOI: 10.1051/epjconf/20147100001

(C) Owned by the authors, published by EDP Sciences, 2014

\title{
Measurement of Properties of the Higgs Boson in the WW(Ivlv) Channel using the ATLAS Detector
}

\author{
H. AbouZeid ${ }^{1, a}$, On behalf of the ATLAS Collaboration \\ ${ }^{1}$ Department of Physics, University of Toronto, 60 St. George St., Toronto, Ontario, M5S 1A7, Canada
}

\begin{abstract}
Measurements are presented of the newly discovered Higgs-like boson in the $W W^{(*)}$ leptonic decay channel, $H \rightarrow W W^{(*)} \rightarrow l v l v$ using data from the ATLAS detector. These results are based on the full $p p$ dataset provided by the Large Hadron Collider (LHC) at CERN, corresponding to an integrated luminosity of $25 \mathrm{fb}^{-1}$ at centre-of-mass energies of $\sqrt{s}=7 \mathrm{TeV}$ and $\sqrt{s}=8 \mathrm{TeV}$. Measurements of the coupling strength, spinparity and evidence for vector boson fusion for the new boson are reported. All measurements of the spin-parity and couplings are consistent with the Higgs boson predicted by the Standard Model.
\end{abstract}

\section{Introduction}

The discovery of a new boson by the ATLAS [1] and CMS collaborations on July 4th, 2012 created great excitement in the field of particle physics. However, precision measurements of the coupling, the production rates and the spin-parity of the new particle are needed to determine whether this is the Higgs boson predicted by the Standard Model (SM), or a more exotic Higgs from a theory beyond the Standard Model.

Since the discovery, ATLAS has more than doubled the dataset used to make the initial observation of the Higgs-like boson. With the increased sensitivity gained by the higher statistics, and tuning an analysis to a specific mass point, measurements of the properties of the new boson are possible. In this contribution an overview of both the analysis determining the coupling measurement and the analysis to determine the spin of the new boson are presented. While these analyses are closely related, they are separated into different sections to make the differences clear.

\section{$2 H \rightarrow W W^{(*)} \rightarrow l v l v$}

One of the main features of the $H \rightarrow W W^{(*)}$ decay channels is the high branching ratio for a large range of hypothetical Higgs masses, making it a very desirable channel for Higgs searches. At $125.5 \mathrm{GeV}$, the mass measured by ATLAS [2], the $W W$ channel has the highest branching fraction after $H \rightarrow b \bar{b}$ decays. While the high branching ratio of this channel make it very attractive for Higgs searches, a mass peak cannot be reconstructed in this analysis, because of the two neutrinos that escape ATLAS without detection and whose only signature is missing energy $\left(E_{\mathrm{T}}^{\mathrm{miss}}\right)$. Only the $W W^{(*)}$

ae-mail: abouzeid@physics.utoronto.ca

This is an Open Access article distributed under the terms of the Creative Commons Attribution License 2.0, which permits unrestricted use, distribution, and reproduction in any medium, provided the original work is properly cited. 


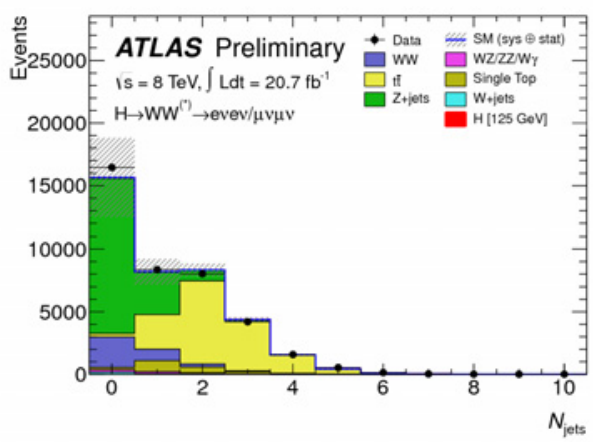

(a)

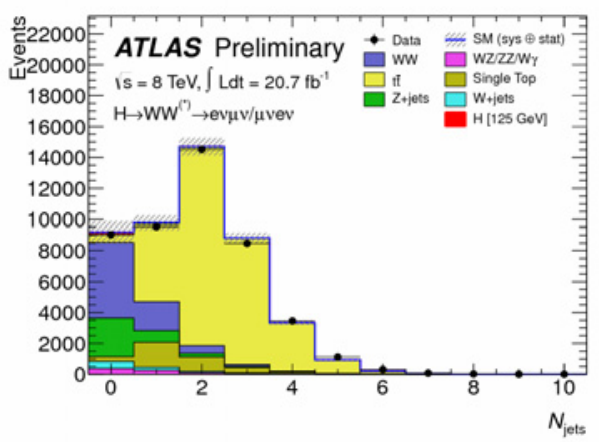

(b)

Figure 1: Signal and background events binned in jet multiplicity for events in $8 \mathrm{TeV}$ data. The plots are shown for the (a) SF and (b) DF channels after pre-selection and $E_{\mathrm{T} \text {,rel }}^{\text {miss }}>25 \mathrm{GeV}$ and $>45 \mathrm{GeV}$, respectively [3].

dilepton final state, where $l=e, \mu$ is discussed here. While optimizations are made for only these two lepton final states, there is a non-zero (but small) contribution to the signal from leptonic tau decays. The final leptonic states are separated into two categories; same flavour (SF, $W W^{(*)} \rightarrow$ evev/ $\mu \nu \mu \nu$ ), and different flavour (DF, $W W^{(*)} \rightarrow e v \mu v / \mu v e v$ ). Each of these four channels have a different background composition, and are separated into different signal regions, with different cuts applied, in order to maximize sensitivity. Of particular importance is the high Drell-Yan background associated with the SF channels, leading to the higher sensitivity in the DF channels.

SM Higgs production at the LHC is dominated by gluon fusion $(\mathrm{ggF})$, calculated with Standard Model predictions to contribute $\approx 87 \%$ to the total Higgs production at $125 \mathrm{GeV}$. Vector boson fusion $(\mathrm{VBF})$ production contributes an order of magnitude less to the total production at $\approx 7 \%$, but is topologically distinct from the ggF mode (and many backgrounds) as a result of the presence of two very forward, opposite hemisphere, high- $p_{\mathrm{T}}$ jets. In order to further maximize sensitivity, the signal region in this analysis is separated into two more categories (in addition to the SF/DF channels) based on the production mode. Events with $\geq 2$ jets are classified as VBF, while all others are classified as ggF, with extra cuts being applied in the VBF case to take advantage of the unique topological signature (see Section 4.2). Both $t \bar{t}$-fusion $(\mathrm{ttH})$ and associated production $(\mathrm{VH})$ are also Higgs production mechanisms accessible at the LHC, but have lower production rates than either ggF or $\operatorname{VBF}(\approx 5 \%, 1 \%$ respectively) and are not discussed here.

\section{Background Treatment}

The dominant backgrounds for the $W W$ analysis are $Z+$ jets, $W W, W+$ jets and top-quark production (which includes both $t \bar{t}$ and single top). Figure 1 shows the relative size of these backgrounds as a function of jet multiplicity, with Figure 1a showing the background contributions in the SF channel and Figure 1b showing the contributions in the DF channel.

The top, $Z / \gamma \rightarrow \tau \tau$ and $W W$ backgrounds are taken from Monte Carlo (MC) and normalized to the observed rate in a data control region. The $W+$ jets background is estimated from data with corrections applied from MC studies. The diboson backgrounds (with the exception of $W W$ ) are 
all extracted directly from simulation but are cross-checked for agreement with data in their own respective validation region.

The control regions/validation regions in each case are selected to be similar to the signal regions, but often have various selection criteria either reversed, modified or removed in order to obtain a region of phase-space that has a small number of signal events with a pure sample of the relevant background.

\section{Coupling Measurement}

\section{1 ggF Selection}

In the pre-selection, events are selected that have exactly two, high- $p_{\mathrm{T}}$, opposite charge leptons and any number of jets. The hardest (leading) lepton is required to have $p_{\mathrm{T}}>25 \mathrm{GeV}$ while the second lepton (sub-leading) must have $p_{\mathrm{T}}>15 \mathrm{GeV}$. A cut on the invariant mass is also applied $\left(m_{l l}>\right.$ $10 \mathrm{GeV}$ for $\mathrm{DF}$ and $>12 \mathrm{GeV}$ for $\mathrm{SF}$ ). In the SF channel, a requirement that $m_{l l}$ does not fall within $15 \mathrm{GeV}$ of the $Z$-mass is applied to reduce the large Drell-Yan background.

Since the coupling measurement assumes a spin-0 Standard Model Higgs boson, cuts can be applied that take advantage of the specific spin correlations in the final state. As a result the two leptons will be preferentially emitted collinearly. The same can be said for the two neutrinos. The signal events will therefore have high $p_{\mathrm{T}}^{l l}$, missing transverse energy $\left(\mathrm{MET}, E_{\mathrm{T}}^{\mathrm{miss}}\right)$, small $m_{l l}$ and a large azimuthal angle between the leptons and $E_{\mathrm{T}}^{\text {miss }}\left(\Delta \phi_{l l, \mathrm{MET}}\right)$.

While signal events will have high $E_{\mathrm{T}}^{\text {miss }}$, the cut applied for the ggF events is actually made on the variable $E_{\mathrm{T} \text {,rel }}^{\mathrm{miss}}$. It is constructed by taking the product of the $E_{\mathrm{T}}^{\mathrm{miss}}$ vector and a projection factor that is defined as $\sin \left|\Delta \phi_{\text {closest }}\right| . \Delta \phi_{\text {closest }}$ is defined as the azimuthal angle between the $E_{\mathrm{T}}^{\text {miss }}$ vector and the nearest lepton or jet. If $\Delta \phi_{\text {closest }}$ is greater than $\pi / 2$, the projection factor takes the value of unity. In the case of DF events, the $E_{\mathrm{T} \text {,rel }}^{\text {miss }}$ cut is applied at $>25 \mathrm{GeV}$, while the SF events have a cut of $>45 \mathrm{GeV}$. The SF channel also has a cut on $p_{\mathrm{T} \text {,rel }}^{\text {miss }}(>45 \mathrm{GeV})$, which is analogous to the $E_{\mathrm{T} \text {,rel }}^{\text {miss }}$ quantity but uses missing momentum, based on measurements from the tracking detectors, instead of the calorimeters. This quantity gives better response given the high number of interactions per bunch crossing at the LHC (known as pile-up), and allows better modelling of the missing energy, which is of particular importance in the SF channel.

Once all cuts have been applied, the final signal regions are defined. For both SF and DF channels the signal region is defined as events with $m_{l l}<50 \mathrm{GeV}$. The signal region for DF events is split into two, with one having $m_{l l}<30 \mathrm{GeV}$, and the other having $m_{l l}>30 \mathrm{GeV}$. The splitting of the signal region in the DF channel takes advantage of the different signal/background ratio and background composition in the two regions.

\subsection{VBF Selection}

To take advantage of the unique VBF event topologies, further cuts are applied to the events in the $\geq 2$ jet bin (in addition to those mentioned in Section 4.1). Events are required to have 2 tag jets with the requirement that the mass of the two jets satisfies $m_{j j}>500 \mathrm{GeV}$ and are well separated in rapidity, $\Delta y>2.8$. VBF events also have an absence of high- $p_{\mathrm{T}}$ jet activity in the central region; events that have an extra jet in the central region of the detector are therefore vetoed. The transverse momentum is also required to be well balanced, $\left|\Sigma \mathbf{p}_{\mathrm{T}}\right|<45 \mathrm{GeV}\left(\left|\Sigma \mathbf{p}_{\mathrm{T}}\right|=\mathbf{p}_{\mathrm{T}}^{\mathrm{jj}}+\mathbf{p}_{\mathrm{T}}^{11}+\mathbf{E}_{\mathrm{T}}^{\mathrm{miss}}\right)$. Finally, because of the large top-quark background in the VBF bin, all events with a $b$-jet are vetoed. 


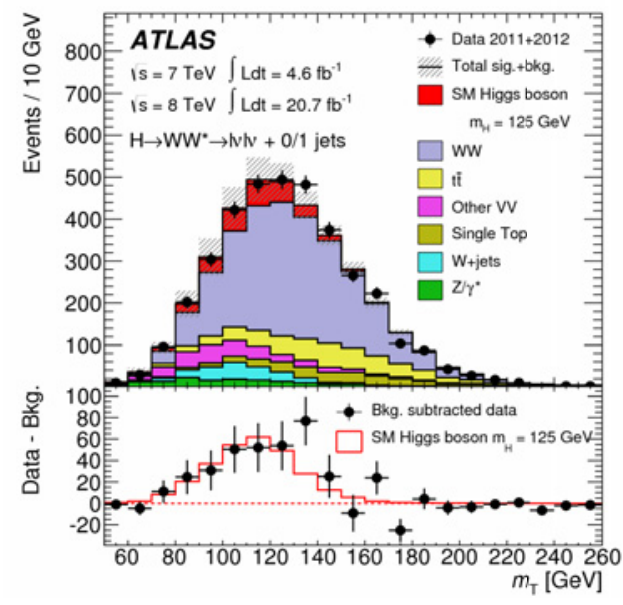

(a)

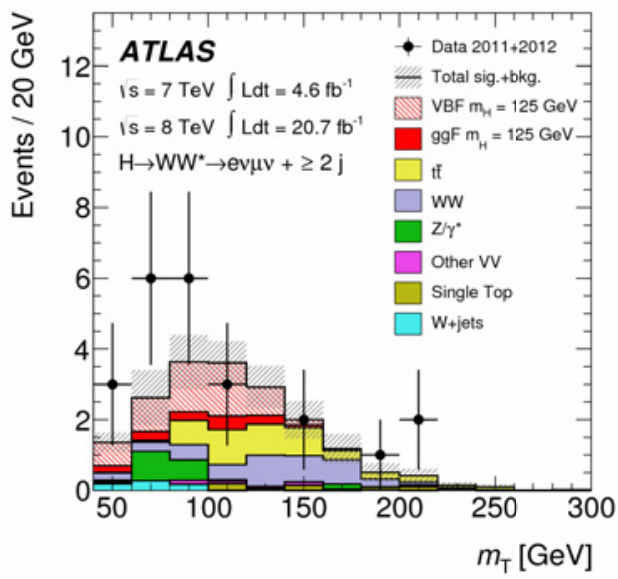

(b)

Figure 2: The transverse mass distributions for events passing the full selection for final states with (a) $\leq 1$ jets and (b) $\geq 2$ jets. The shaded region shows the total uncertainty on the number of events, including experimental, systematic and theoretical sources [2].

While the ggF selection (Section 4.1) uses the kinematic variable $E_{\mathrm{T}, \mathrm{rel}}^{\mathrm{miss}}$, the VBF selection instead uses an $E_{\mathrm{T}}^{\text {miss }}$ cut (DF: $>20 \mathrm{GeV} \mathrm{SF}:>45 \mathrm{GeV}$ ). The VBF bin has more jet activity in the events, which results in the degraded performance of $E_{\mathrm{T}, \text { rel }}^{\mathrm{miss}}$.

The final signal region in the VBF bin is also defined by the invariant mass of the leptons and requires $m_{l l}<60 \mathrm{GeV}$.

\subsection{Results}

Once all the cuts have been applied, the transverse mass of each remaining event is calculated. The definition of transverse mass is given as;

$$
m_{\mathrm{T}}=\sqrt{\left(E_{\mathrm{T}}^{l l}+E_{\mathrm{T}}^{\mathrm{miss}}\right)^{2}-\left|p_{\mathrm{T}}^{l l}+E_{\mathrm{T}}^{\mathrm{miss}}\right|^{2}}
$$

where $E_{\mathrm{T}}^{l l}=\sqrt{\left|p_{\mathrm{T}}^{l l}\right|^{2}+m_{l l}^{2}}$. The transverse mass distributions for the ggF and VBF production channels can be found in Figure 2. While the number of events in the VBF bin is much smaller compared to $\mathrm{ggF}$ (because of the lower production rate) it can be seen that the signal to background ratio is much higher.

Statistical analysis of the data uses a likelihood function $(\mathcal{L})$ comprised of a product of Poisson distributions for each signal and control region. The signal region has an additional free term, $\mu$, which denotes the signal strength. A signal strength of $\mu=0$ indicates no signal, while $\mu=1$ indicates a coupling strength equal to the SM prediction.

The $H \rightarrow W W^{(*)} \rightarrow l v l v$ analysis sees a broad excess of events compared to the background only hypothesis as shown in Figure 3. The greatest deviation from the background only hypothesis is $4.1 \sigma$ at $140 \mathrm{GeV}$, while at $125 \mathrm{GeV}$ the analysis deviates from the background only hypothesis by $3.8 \sigma$ 


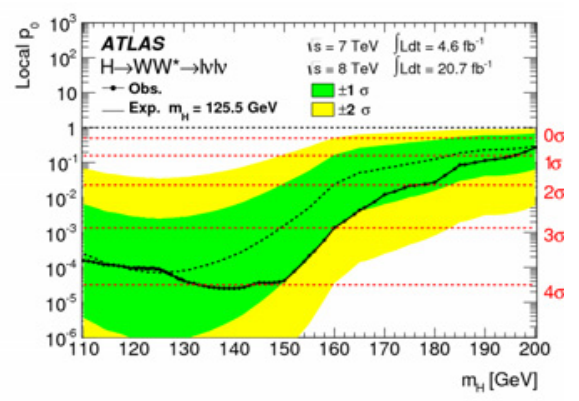

Figure 3: The expected and observed local $p_{0}$ for different $m_{H}$ values [2].

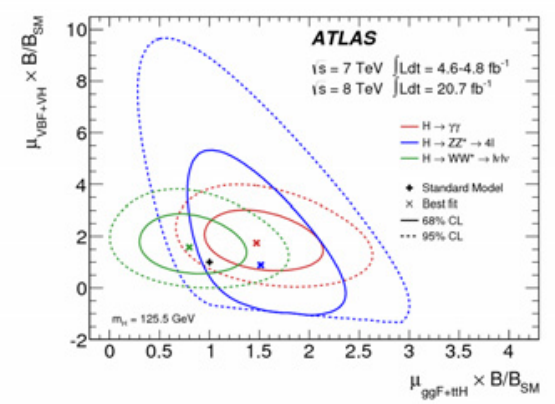

Figure 4: Likelihood contours for the $H \rightarrow$ $\gamma \gamma, H \rightarrow Z Z^{(*)} \rightarrow 4 l$ and $H \rightarrow W W^{(*)} \rightarrow l v l v$ channels are shown in the $\mu_{\mathrm{ggF}+\mathrm{ttH}} \times B / B_{\mathrm{SM}}$, $\mu_{\mathrm{VBF}+\mathrm{VH}} \times B / B_{\mathrm{SM}}$ plane [2].

compared to $3.8 \sigma$ expected given a $125 \mathrm{GeV}$ SM Higgs boson. The signal strength observed by this analysis across all signal regions and production modes is $\mu_{\mathrm{obs}}=0.91 \pm 0.21$ (stat.) \pm 0.21 (syst.). The signal strength can also be extracted individually for the ggF and VBF production modes (while accounting for the fact that there is a contamination of $\mathrm{VBF}$ events in the $\mathrm{ggF}$ bin, and vice versa). For $\mathrm{ggF}$ the signal strength is determined to be, $\mu_{\mathrm{obs}, \mathrm{ggF}}=0.82 \pm 0.32$, while for $\mathrm{VBF}, \mu_{\mathrm{obs}, \mathrm{VBF}}=1.7 \pm 0.7$. All values for $\mu_{\mathrm{obs}}$ are fully consistent with the SM hypothesis of $\mu=1$. These results can be seen in Figure 4 along with the results from the ATLAS $H \rightarrow \gamma \gamma$ and $H \rightarrow Z Z^{(*)} \rightarrow 4 l$ analyses.

\section{Spin Measurement}

Studies were performed to determine the spin-parity $J^{P}$ of the new boson using the data collected in $2012\left(21 \mathrm{fb}^{-1}\right)$. The Standard Model hypothesis of $0^{+}$was compared to alternative hypotheses of $1^{+}, 1^{-}, 2^{+}$in the $W W$ channel $[4,5]$. The coupling analysis (discussed in Section 4) gains sensitivity by assuming a Standard Model like Higgs boson $\left(0^{+}\right)$, and therefore cuts that take advantage of the spin correlations were relaxed for the spin analysis. This analysis only considers the 0 -jet DF events in order to maximize sensitivity.

The spin-2 model studied in this analysis was based on a simple model with a graviton-like tensor and minimal couplings (denoted $2_{m}^{+}$). Since the relative fraction of the production mechanisms, $g g \rightarrow$ $H$ and $q \bar{q} \rightarrow H$ is unknown $\left(f_{q \bar{q}}\right)$, the case of $J^{P}=2^{+}$was performed with five different values of $f_{q \bar{q}}$ with values ranging from $0 \%$ to $100 \%$ in $25 \%$ intervals.

In order to discriminate between the various $J^{P}$ hypotheses and the SM $0^{+}$hypothesis, a 2-D kinematic shape fit was used. The discriminants in the fit were outputs of two different Boosted Decision Trees (BDT). One BDT was trained on the SM hypothesis, while the other was trained on one of the alternate hypotheses. Four variables were fed as inputs into the BDT for training: the invariant mass, azimuthal separation and transverse momentum of the dilepton system $\left(m_{l l}, \Delta \phi_{l l}\right.$, $\left.p_{\mathrm{T}}^{l l}\right)$ and the transverse mass $\left(m_{\mathrm{T}}\right)$. Of these discriminating variables, the most sensitive are $\Delta \phi_{l l}$ and $m_{l l}$, which are shown in Figure 5. The compatibility of the data with the SM/alternate hypothesis is estimated by $q$ and given by;

$$
q=\log \frac{\mathcal{L}\left(H_{0^{+}}\right)}{\mathcal{L}\left(H_{J^{P}}\right)}
$$




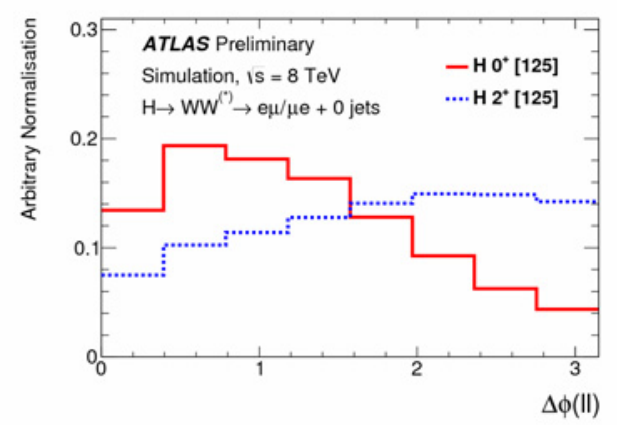

(a)

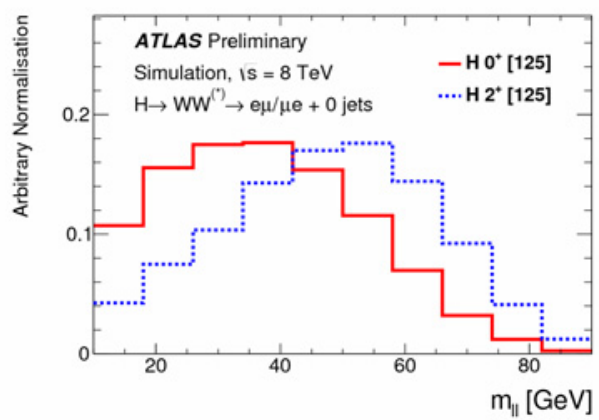

(b)

Figure 5: Distributions of (a) $\Delta \phi_{l l}$ and (b) $m_{l l}$ for $J^{P}=0^{+}$and $J^{P}=2_{m}^{+}$hypotheses. Both distributions are normalised to unit area [5].

The likelihoods in both the numerator and denominator (denoting the two $J^{P}$ hypotheses) are maximized. It is the value of $q$ that determines the CL for the exclusion of the given alternate hypothesis.

\subsection{Results}

The results for the spin $2^{+}$model are shown in Figure 6 . For all values of $f_{q \bar{q}}$ the spin $0^{+}$hypothesis is preferred. The results from the $W W$ channel alone exclude the $J^{P}=2^{+}$hypothesis at confidence levels (CL) varying from $>95 \%$ to $>99 \%$ for $f_{q \bar{q}}=0 \%$ and $f_{q \bar{q}}=100 \%$, respectively. When combined with other ATLAS search channels, the spin $2^{+}$hypothesis is excluded at $>99 \%$ CL for all values of $f_{q \bar{q}}[4]$.

The CLs for the other $J^{P}$ hypothesis are summarized in Figure 7. These results are not from the $W W$ channel alone, but also include two other Higgs search channels $\left(H \rightarrow \gamma \gamma, H \rightarrow Z Z^{(*)}\right)$. It should be noted that only the $H \rightarrow Z Z^{(*)}$ analysis contributed to the exclusion of the $J^{P}=0^{-}$hypothesis. For the other spin-parity hypotheses, the Standard Model prediction of $J^{P}=0^{+}$is preferred with the alternative hypotheses being excluded at $>99 \%$ CL.

\section{Conclusions}

Measurements of the signal strength of the Higgs boson in the production mechanisms ggF and VBF are reported for the $H \rightarrow W W^{(*)}$ channel. In both cases, the results are fully consistent with the Standard Model prediction. In addition, the spin-parity state of a Standard Model Higgs $\left(J^{P}=0^{+}\right)$ tested against multiple other spin-parity hypotheses $\left(J^{P}=1^{+}, 1^{-}\right.$and $\left.2^{+}\right)$. In all cases, the Standard Model spin-parity is preferred, and the alternate hypotheses are excluded at $>99 \%$ CL.

\section{Acknowledgements}

The author would like to thank the generous financial support of the Natural Sciences and Engineering Council of Canada (NSERC) that helped make this work possible. 


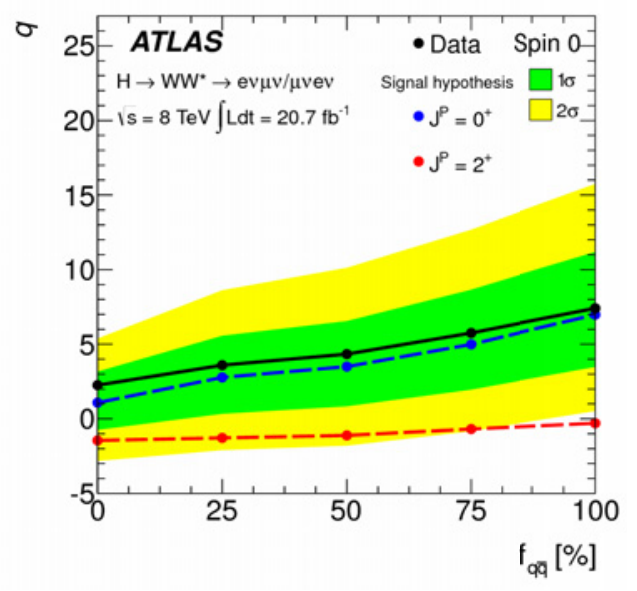

Figure 6: Values of the test statistic $q$ versus the fraction $f_{q \bar{q}}$ [4].

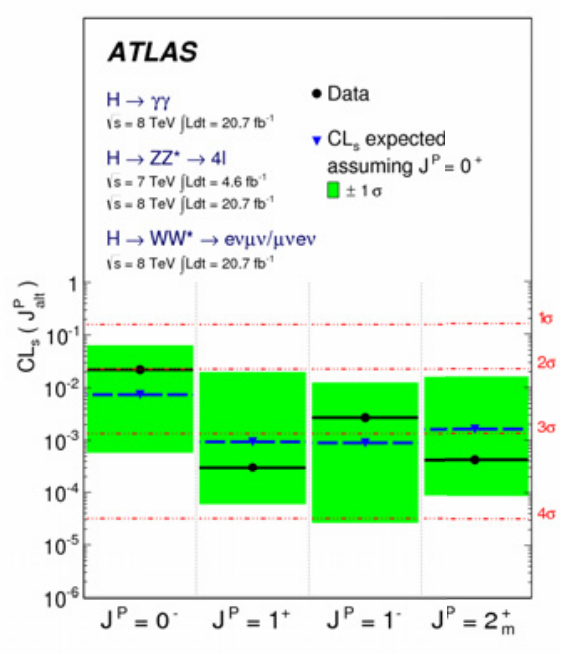

Figure 7: Observed and expected CLs for various $J_{\text {alt }}^{P}$ hypotheses assuming a SM Higgs boson with $J^{P}=0^{+}[4]$.

\section{References}

[1] ATLAS Collaboration, JINST 3S08001 (2008)

[2] ATLAS Collaboration, Physics Letters B, 726, 88-119 (2013)

[3] ATLAS Collaboration, ATLAS-CONF-2013-030, http://cds.cern.ch/record/1527126

[4] ATLAS Collaboration, Physics Letters B, 726, 120-144 (2013)

[5] ATLAS Collaboration, ATLAS-CONF-2013-031, http://cds.cern.ch/record/1527127 